\title{
The High-Strain Rate Loading of Structural Biological Materials
}

\author{
W.G. PROUD, T.-T.N. NGUYEN, C. BO, B.J. BUTLER, R.L. BODDY, A. WILLIAMS, \\ S. MASOUROS, and K.A. BROWN
}

\begin{abstract}
The human body can be subjected to violent acceleration as a result of explosion caused by military ordinance or accident. Blast waves cause injury and blunt trauma can be produced by violent impact of objects against the human body. The long-term clinical manifestations of blast injury can be significantly different in nature and extent to those suffering less aggressive insult. Similarly, the damage seen in lower limbs from those injured in explosion incidents is in general more severe than those falling from height. These phenomena increase the need for knowledge of the short- and long-term effect of transient mechanical loading to the biological structures of the human body. This paper gives an overview of some of the results of collaborative investigation into blast injury. The requirement for time-resolved data, appropriate mechanical modeling, materials characterization and biological effects is presented. The use of a range of loading platforms, universal testing machines, drop weights, Hopkinson bars, and bespoke traumatic injury simulators are given.
\end{abstract}

DOI: $10.1007 / \mathrm{s} 11661-015-2975-4$

(C) The Minerals, Metals \& Materials Society and ASM International 2015

\section{INTRODUCTION}

RECENT military conflicts in Iraq and Afghanistan have resulted in a rising incidence of injuries from blast and blunt trauma. ${ }^{[1,2]}$ Improved medical care has resulted in higher survival rates from such injuries. The injury patterns seen are quite different from those resulting from civilian incidents such as falling from height or traffic accidents. ${ }^{[3]}$ Fundamental reasons for this difference include loading rates that are significantly higher in blast and high-speed blunt trauma scenarios, and the presence of a blast wave.

A description of the physical processes of blast and explosion has been presented by the author, ${ }^{[4]}$ indicating the levels of force and acceleration involved. Researchers have found that stress and pressure required to produce hearing, intestines, larynx, and lung damage are

W.G. PROUD, Reader, and T.-T.N. NGUYEN and C. BO, PhD Students, are with The Royal British Legion Centre for Blast Injury Studies, Imperial College London, London, U.K., and also with the Institute of Shock Physics, Imperial College London. Contact e-mail: w.proud@imperial.ac.uk B.J. BUTLER, PhD Student, and R.L. BODDY, Postdoctoral Researcher, are with The Royal British Legion Centre for Blast Injury Studies, Imperial College London, and also with the Surfaces, Microstructure and Fracture Group, Cavendish Laboratory, University of Cambridge, Cambridge, U.K. A. WILLIAMS, Professor, is with the Department of Veterinary Medicine, Veterinary School, University of Cambridge. S. MASOUROS, Lecturer, is with The Royal British Legion Centre for Blast Injury Studies, Imperial College London, and also with the Department of Bioengineering, Imperial College London. K.A. BROWN, Senior Research Fellow, is with The Royal British Legion Centre for Blast Injury Studies, Imperial College London, and also with the Surfaces, Microstructure and Fracture Group, Cavendish Laboratory, University of Cambridge, and also with the Department of Chemistry, The University of Texas at Austin, Austin, TX. Manuscript submitted September 1, 2014.

Article published online July 14, 2015 relatively modest, compared to those loads commonly used to study engineering structures. ${ }^{[5]}$ Conversely, the loading rate from blasts are much higher than usually encountered in many standard testing regimes. Blast produces specific injury profiles and causes of fatality [e.g., References 3,6], along with long-term clinical manifestations such as traumatic brain injury ${ }^{[7]}$ and heterotopic ossification. ${ }^{[8]}$

The overall research strategy adopted by the authors has been presented in a briefing ${ }^{[9]}$ and a discussion of the use of high-rate loading platforms appropriate to blast was recently presented. ${ }^{[10]}$ This paper will outline two aspects of this research in greater detail. First, the development and use of appropriate experimental platforms to apply the relevant range of loading rate to biological materials. After this, selected results illustrating the response of tissues, organs, and structures at blast-relevant loading rates will be presented.

\section{BLAST LOADING-SHOCK TUBE DEVELOPMENT}

The shock tube is a versatile, simple device invented in 1899 , which is able to generate a range of well-defined pressure pulses. It has been used to study blast and shock wave structures in areas such as mine safety, hydrodynamics, geophysics, chemical kinetics, and plasma physics. ${ }^{[11}$ The most important in this study is its ability to create controlled blast waves in the laboratory environment.

A blast wave generated by the violent expansion of product gases from an explosion consists of a sharp pressure rise followed by a more gradual decrease in pressure to levels below that of atmospheric pressure, 
eventually the pressure returns to atmospheric pressure after a series of smaller oscillations. The Friedlander waveform (Figure 1(a)) is a classic representation of a pressure history ${ }^{[12]}$ observed from blast, where it can expand freely in an open space with no reflecting surfaces present. ${ }^{[13,14]}$ However, in the case of an enclosed space, e.g., urban environments or inside vehicles, the waveform is more complex (Figure 1(b)) with multiple overlapping waves caused by reflection of the incident wave off surrounding surfaces. ${ }^{[13]}$

The shock tube in the Centre for Blast Injury Studies at Imperial College London is $3.8 \mathrm{~m}$ long, with an internal diameter of $59 \pm 1 \mathrm{~mm}$ (Figure 2). Compressed air is used to pressurize the driver section, while the driven section remains at atmospheric pressure. The system may be used in a single- or double-diaphragm mode. Double-diaphragm mode is shown in Figure 2 and this is used to produce blast waves with a sharp initial pressure rise. For double-diaphragm operation the system is pressurized so that the pressure between the diaphragms is half of that in the driver section. When the pressure between the diaphragms is dropped the rear diaphragm deforms and tears. This releases the pressure rapidly which strikes the front membrane and rapidly breaks it. Using aluminum or Mylar ${ }^{\circledR}$ diaphragms of varying thickness, blast waves with magnitudes between 0.5 and 17.0 bars are produced, equivalent to the output from a detonation of $20 \mathrm{~kg}$ TNT at distances of 1.2 to $12 \mathrm{~m} .{ }^{[16]}$ Nguyen et al. ${ }^{[15]}$ showed that the doublediaphragm configuration gave more control over the output as it allows the diaphragm to be burst at a specific pressure and timing.

If the minimum driving volume is required the driver section is replaced with a blanking flange. This gives a single-diaphragm configuration where increasing the

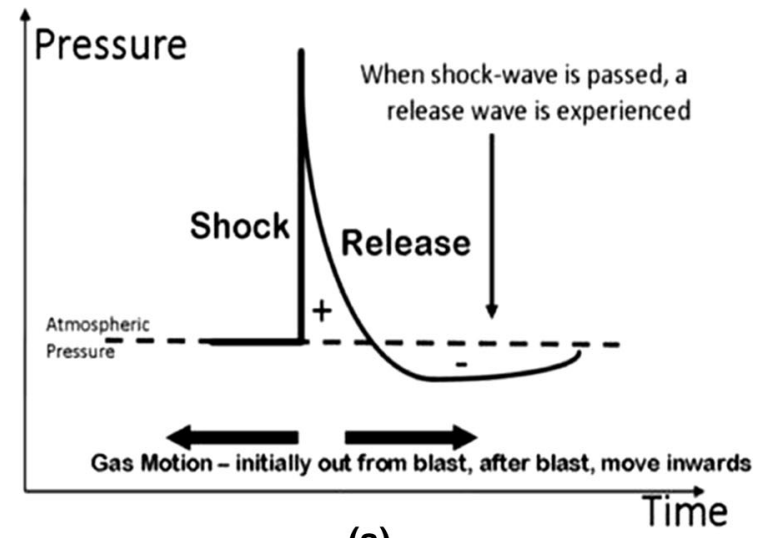

(a) pressure behind the diaphragm causes it to rupture. In this case, the burst pressure is fixed by the strength of the diaphragm giving a much coarser control of blast pressure compared to the double-diaphragm system.

As the blast wave propagates down the shock tube, its evolution can be monitored using several piezoelectric transducers (Dytran 2300V1) oriented laterally at various positions along the tube, and on-axis at the outlet of the tube.

The duration of the pressure pulse can be controlled in a relatively simple manner. The sudden rupture of the diaphragm releases the high-pressure gas into the lowpressure section, forming a blast wave. Simultaneously, a rarefaction wave is also created, which travels back into the high-pressure region. The time taken for this release to reach the rear of the drivers section controls the duration of the blast pulse.

In addition, the shock tube can be used in both closed and open configurations. Figure 2 shows the closed configuration. The closed configuration gives rise to multiple reflected shock waves reproducing scenarios with reflecting walls or barriers; a typical output is shown in Figure 3(a). In the open configuration, the outlet flange is removed, and the head-on sensor is mounted on a bullnose instead of the outlet flange.

Polyethylene inserts are used to reduce the length of the driving gas in the driver section, Nguyen et al. ${ }^{[15]}$ showed the blast duration changes linearly with the length of the driving volume without significantly affecting the magnitude. The combination of small driving volume and open configuration produces blast waves of the Friedlander waveform (Figure 3(b)). ${ }^{[14]}$

Perforated sheets and granular beds can be inserted into the driven section to reproduce blast attenuation effects. $^{[17]}$ The magnitude of the initial pulse can be reduced by decreasing the area of the perforations

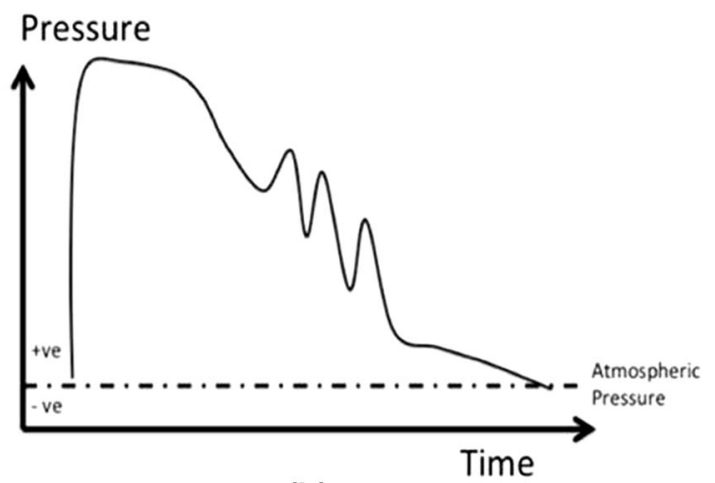

(b)

Fig. 1-(a) The Friedlander waveform representation of blast. (b) Schematic representation of a blast wave inside a vehicle ${ }^{[12,13]}$.

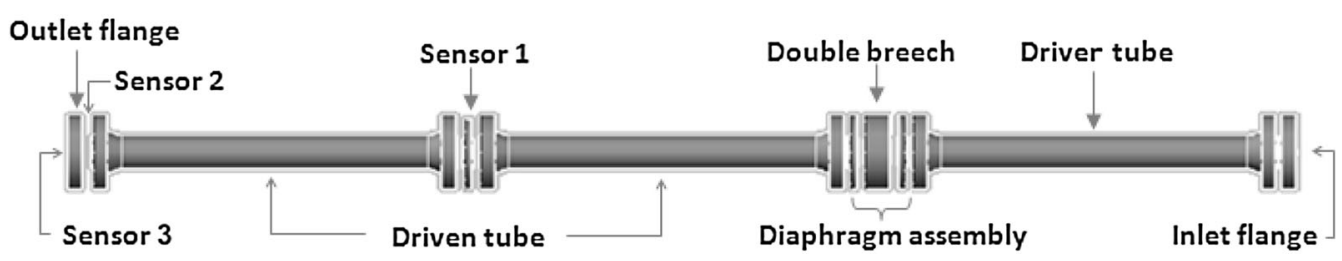

Fig. 2-Schematic of the shock tube at the Centre for Blast Injury Studies. 


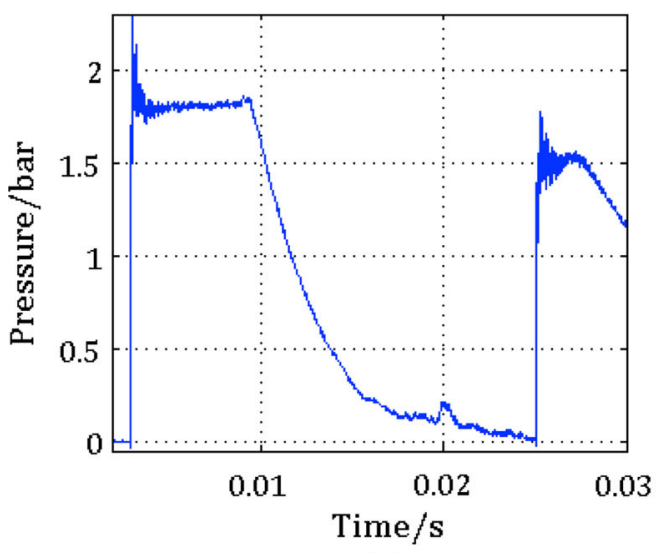

(a)

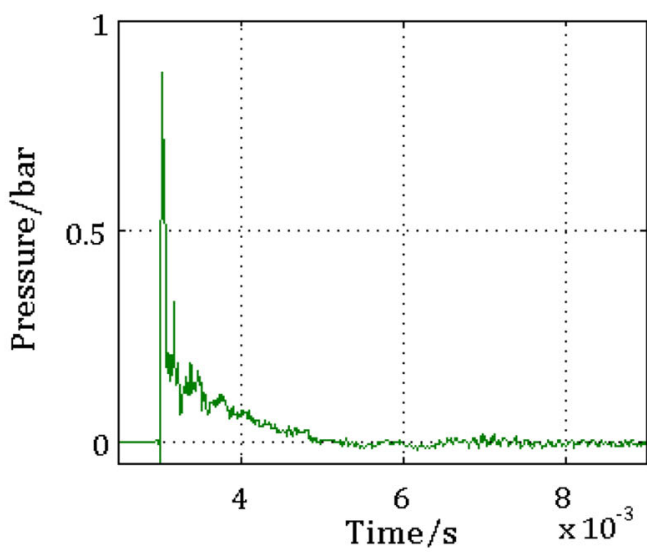

(b)

Fig. 3-Pressure profile recorded by the head-on sensor 3 for $(a)$ Full driving volume in closed configuration and (b) 10 pct of driving volume in open configuration. The diaphragms used were $40 \mu \mathrm{m}$ annealed aluminum foil.

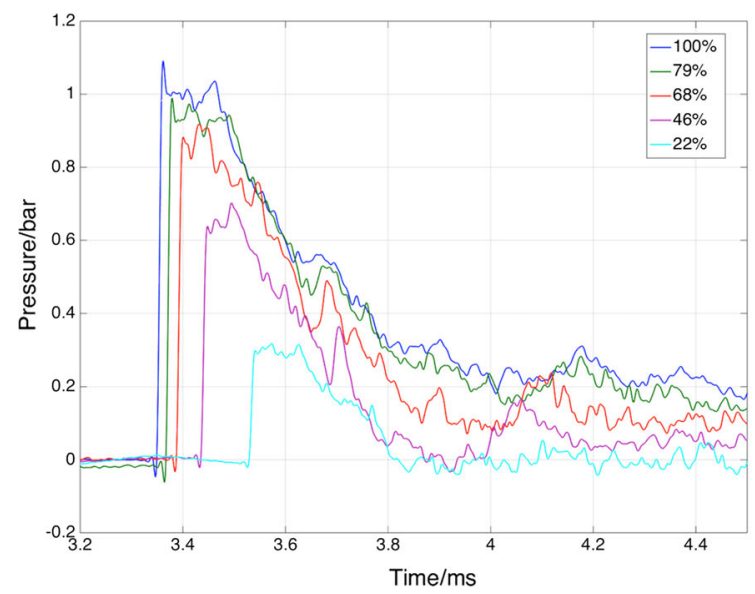

(a)

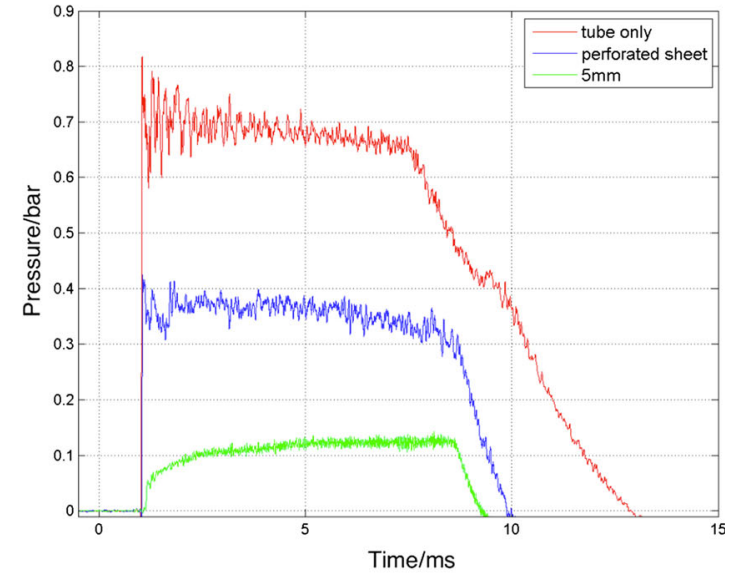

(b)

Fig. 4-(a) Transmitted pressures for various open areas of perforated galvanized steel sheet and $(b)$ pressure profiles showing effects of granular beds. Traces were recorded by lateral pressure sensor 2, diaphragms used were $50 \mu \mathrm{m}$ Mylar ${ }^{\circledR[17]}$.

(Figure 4(a)). Granular beds not only attenuate the blast but also transform the initial rise into a ramped structure (Figure 5(b))..$^{[17]}$

This ability to produce a range of pressure profiles, control pulse height, duration, and shape allows the effect of different mitigants to be assessed and this range of blast pulses can be used to investigate human tissue response to understand the levels of force which result in varying levels of injury.

\section{BLUNT IMPACT LOADING-ANUBIS}

Battlefield data suggest that, for casualties of IEDs, $\sim 92$ pct of the mounted (in-vehicle) personnel sustain disabling injuries to the lower extremities and $\sim 69 \mathrm{pct}$ for dismounted (on-foot) personnel. ${ }^{[3]}$ The injuries seen for personnel mounted or dismounted were characterized by violent axial loading. ${ }^{[18]}$ To understand the injury mechanisms and develop better mitigation strategies, a traumatic injury simulator (AnUBIS) was developed. ${ }^{[19]}$ This replicates, in the laboratory, a wide range of possible insults to the floor of a vehicle and allows for various leg postures to be studied. This increases the understanding of the load-transfer mechanisms, as well as allowing the evaluation of mitigation systems. This system has also been used to quantify the ability of current and new surrogates to replicate human behavior.

The fundamental arrangement is shown in Figure 5(a). The system is effectively a short-barreled vertical firing gas gun. A test specimen is placed on the central metal plate, this plate is retained in position by a retaining pin. The pressure vessel is filled until the shear pin breaks launching the plate. As the plate moves it is decelerated by the surrounding spring-loaded levers (Figure 5(b)). This gives an initial velocity of up to $\sim 20 \mathrm{~m} / \mathrm{s}$ which decreases dramatically coming to rest over a total travel distance of $30 \mathrm{~cm}$. This allows legs and other structural elements of the human body to be assessed in the range of velocities and acceleration seen in combat situations (see Section IV-D). 


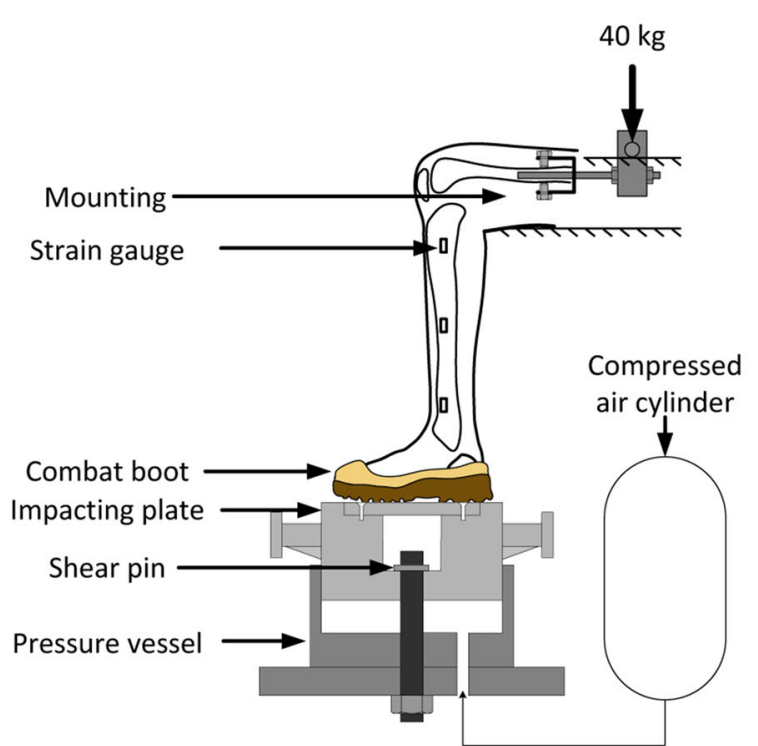

(a)

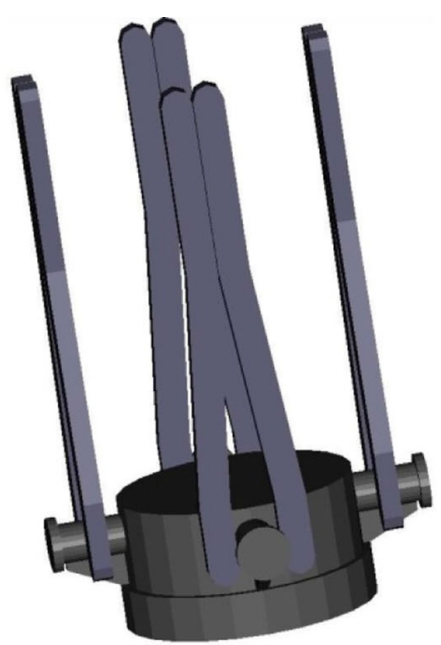

(b)

Fig. 5-Schematic of the Anubis System. (a) The pressure system arrangement with leg in place. (b) The lateral, spring-loaded levers around the impacting plate which decelerate the impacting plate.

\section{BIOLOGICAL MATERIALS UNDER DYNAMIC LOADING}

The relationship between blast stimulus (pressure, duration), and the level of injury to specific areas of the body is not well established. In the 1960's, Richmond et $a l^{[20]}$ have reported lethality curves for different animals as a function of overpressure and duration for open-air experiments. Based on these studies, a blast of $400 \mathrm{~ms}$ and peak pressure of 600 psi ( 40 bar) would cause 99 pct lethality for mammals of $70 \mathrm{~kg}$ weight. In a more recent study, Bass et al. ${ }^{[21]}$ analyzed data from more than 2550 large animal experiments and defined new blast injury thresholds for short-duration blasts $(<30 \mathrm{~ms})$ in the order of 1 to $2 \mathrm{MPa}$ peak pressure. Although these studies give a good indication of the pressure-time regime associated with blast injuries, they lack characterization of the pressures and strains developed inside the body at different length and time scales.

To develop a deeper understanding of the injury process and endurance limits of the human body it is necessary to study the response of the body across different length and structural scales. In this section, four types of length and material scale are reported, cellular, soft tissue, fibrous tissue, and structural. Of these the cellular level is most extensively reported here, while the structural level gives clear indications of the levels of injury associated with blast. The area of soft and fibrous tissues is being investigated and will be the subject of more extensive discussion in subsequent publications.

\section{A. Cellular Level}

Our studies have initially focused on understanding more about the resilience and functional properties of cells that play significant roles in blast injury. Using a
Split Hopkinson Pressure Bar (SHPB) system, ${ }^{[22,23]}$ fitted a biocompatible confinement chamber (Figure 6), cells in solution or adhered monolayers on coverslips were subjected to a range of pressure pulses.

The chamber is composed of a polycarbonate cylinder with inner O-ring grooves. Two polycarbonate disks with an O-ring groove on the inner side are pushed against the main body by brass locking rings to improve the sealing of the liquid inside the chamber. The chamber is mounted on a compressive SHPB system composed of four bars made from Inconel steel, $12.7 \mathrm{~mm}$ in diameter, and $190 \mathrm{~mm}$ (striker) or $500 \mathrm{~mm}$ (input, output and momentum trap) in length. Liquid samples are inserted using a syringe through $1-\mathrm{mm}$ diameter counter-bored holes, which are then sealed with a nylon screw. Alternatively, coverslips can be placed on a support bar which is then inserted into the chamber. The chamber can then be filled with liquid media using a syringe. The chamber is instrumented with two foil strain gages GFLA-3-350-70 (TechniMeasure, UK) located halfway along the chamber length and diametrically opposite each other in order to measure the circumferential strain.

This chamber was used to study the survivability of mesenchymal stem cells (MSCs), a cell-type identified to be of potential importance in the development of heterotopic ossification, a post-traumatic condition involving the formation of ectopic bone following blast injury. ${ }^{[24,25]}$ MSCs were selectively cultured from the periosteum and bone marrow of $\mathrm{Balb} / \mathrm{c}$ mice. Cell cultures at a concentration of $10^{6}$ cells $/ \mathrm{mL}$ were aliquoted as $800-\mu \mathrm{L}$ samples into $1.5-\mathrm{mL}$ microfuge tubes. Samples (five per condition) were divided into five groups: control, sham (cells that are inserted using a syringe in the chamber and recovered without being subjected to pressure waves), Blast 1 and Blast 2 (cells that are inserted in the chamber and subjected to 

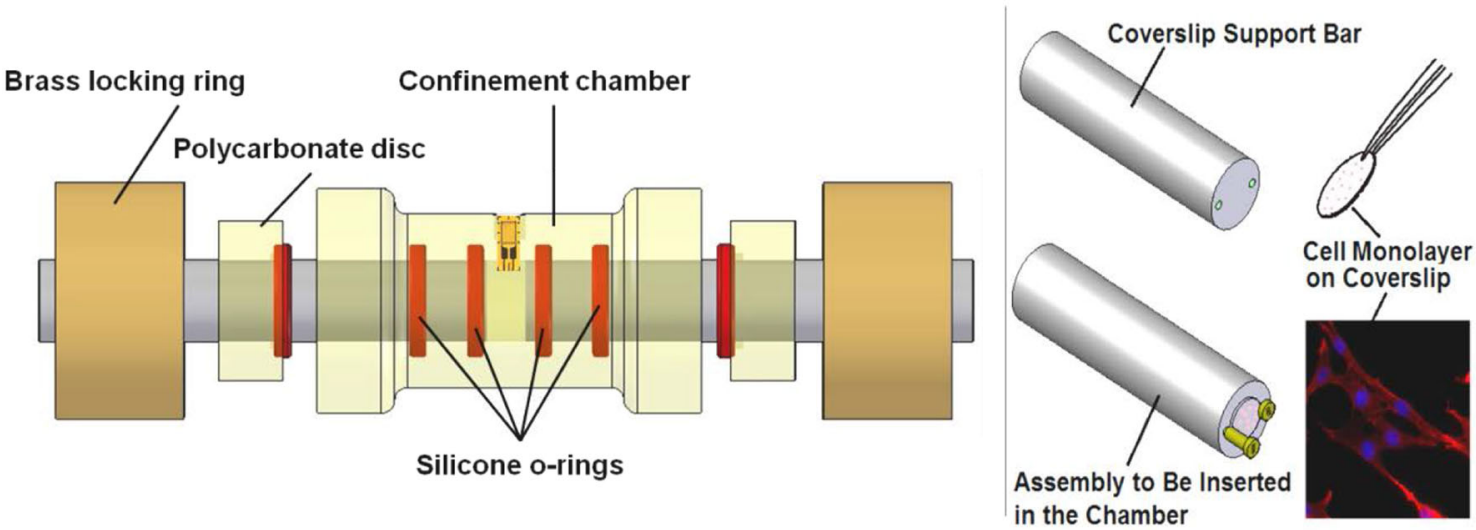

Fig. 6-Experimental assembly for confined SHPB experiments with cells in solution or as monolayers on coverslips. Components of the assembly are labeled and liquid samples are introduced into the confinement chamber through 1-mm bore holes.

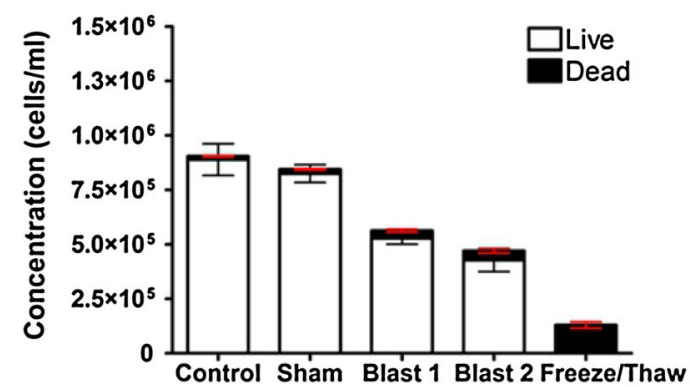

(a)

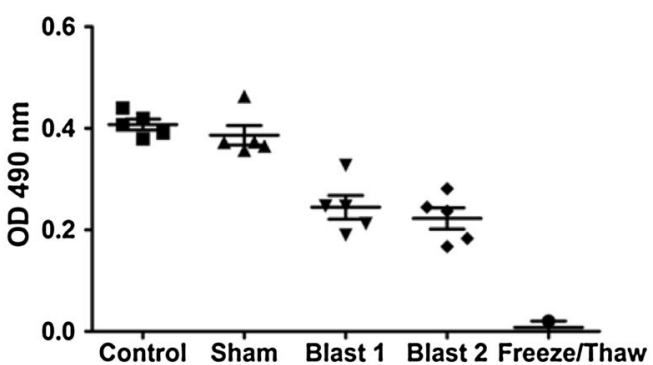

(b)

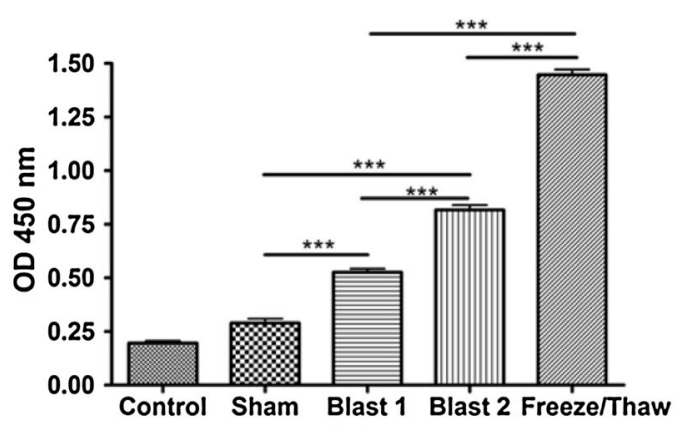

(c)

Fig. 7-Comparisons of MSC survival at two different dynamic pressures, Blast 1 and Blast 2 (described in text). See text also for descriptions of controls (CTL), sham and freeze/thaw samples. (a) Cell concentrations of MSCs determined using the Trypan blue. (b) Cell respiration measured with a MTS colorimetric assay. (c) LDH levels in the supernatants of samples recovered post compression. [***p $<0.001$, one-way analysis of variance (ANOVA) statistical test].

pressure pulses of different magnitudes) and freeze/thaw (samples that are subjected to two series of freeze and thawing procedures to obtain a total cell lysate). Three biological assays were performed to assess the effects of the pressure pulses on MSCs: cell counting with Trypan blue dye, cell viability using a one-step MTS assay (CellTiter96, Promega), and cell damage/lysis assessed on cell-free supernatants using a lactate dehydrogenase (LDH) release assay (ab65393, Abcam).

Survival of MSCs post compression was assessed using both Trypan blue dye to count cells and by measuring the cell respiration levels using the MTS assay. Results suggest that cell survival decreases as a function of the intensity of the pressure pulse (Figures 7(a) and (b)). Cell viability was investigated by measuring the release of LDH into the supernatant of the recovered samples. LDH levels in the samples increased as a function of the intensity of the compression waves applied. These data suggest that cells are lysed due to the subjected mechanical stimulus in the SHPB experiments (Figure 7(c)). Although a correlation between the peak pressure and impulse on cell viability, was observed, other mechanical parameters (i.e., strain rate) and mechanical phenomena (i.e., cavitation) could have contributed to the lysis of cells in suspension, as previously reported in extracorporeal shock wave treat- 


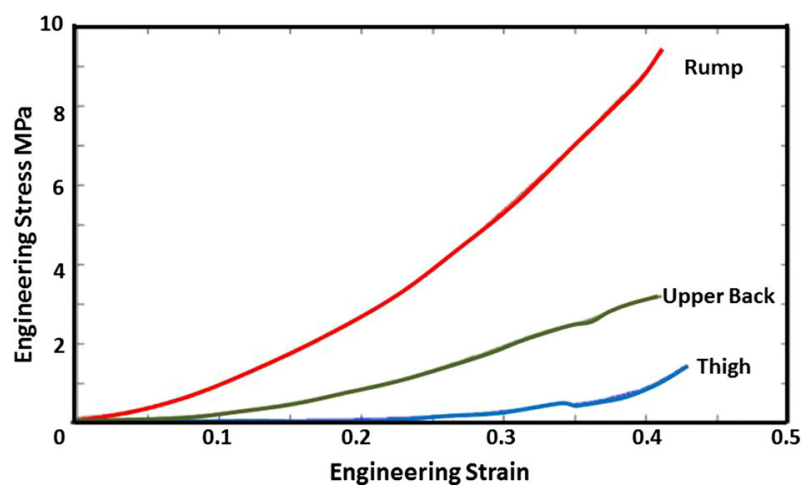

Fig. 8-Comparison of the engineering stress-strain curves for skin taken from different anatomical regions. These were obtained on an Instron testing rig at a strain rate of $1 \mathrm{~s}^{-1}$.

ment and laser shock experiments. ${ }^{[26,27]}$ These parameters are undergoing further study.

\section{B. Soft Tissue Level}

Cellular responses are also dependent upon local tissue environments. Soft tissues associated with skin and respiratory system are often damaged by blast. ${ }^{[28]}$ Characterizing the material properties of these tissues at over a range of loading rates representative of injury conditions will be an important step toward developing biofidelic models for mitigation and biomedical applications. We have initiated comparative studies of the heterogeneous properties of fresh porcine skin harvested from different anatomical regions (rump, upper back, and thigh). To do this, a rectangular sheet was harvested from each anatomical area of interest and the adipose layer was removed from each with a scalpel. Cylindrical specimens, about $8 \mathrm{~mm}$ in diameter, were obtained using a biopsy punch. These specimens were stored in phosphate buffered saline solution at $277 \mathrm{~K}\left(4{ }^{\circ} \mathrm{C}\right)$ until mechanical tests were performed, up to 5 hours postmortem. Prior to testing, each sample was positioned between two microscope slides and the thickness was measured. Compression experiments were performed at different strain rates using an Instron 5566 and a SHPB system. Data obtained at a strain rate of $1.0 \mathrm{~s}^{-1}$ using the Instron are shown in Figure 8. These data illustrate variation in the relative stiffness of these skin samples, reflecting differences in their underlying structure and compositions.

After compression, skin tissue samples were recovered and fixed in formaldehyde for 24 hours. Figure 9 shows optical images of histological sections stained with Masson's trichrome to investigate skin subjected to compression at low- and medium-strain rates using the Instron and SHPB. These images show increased infiltration of dye in the samples subjected to compression compared to the sham. The level of dye infiltration decreases as the applied strain rate of loading increases.

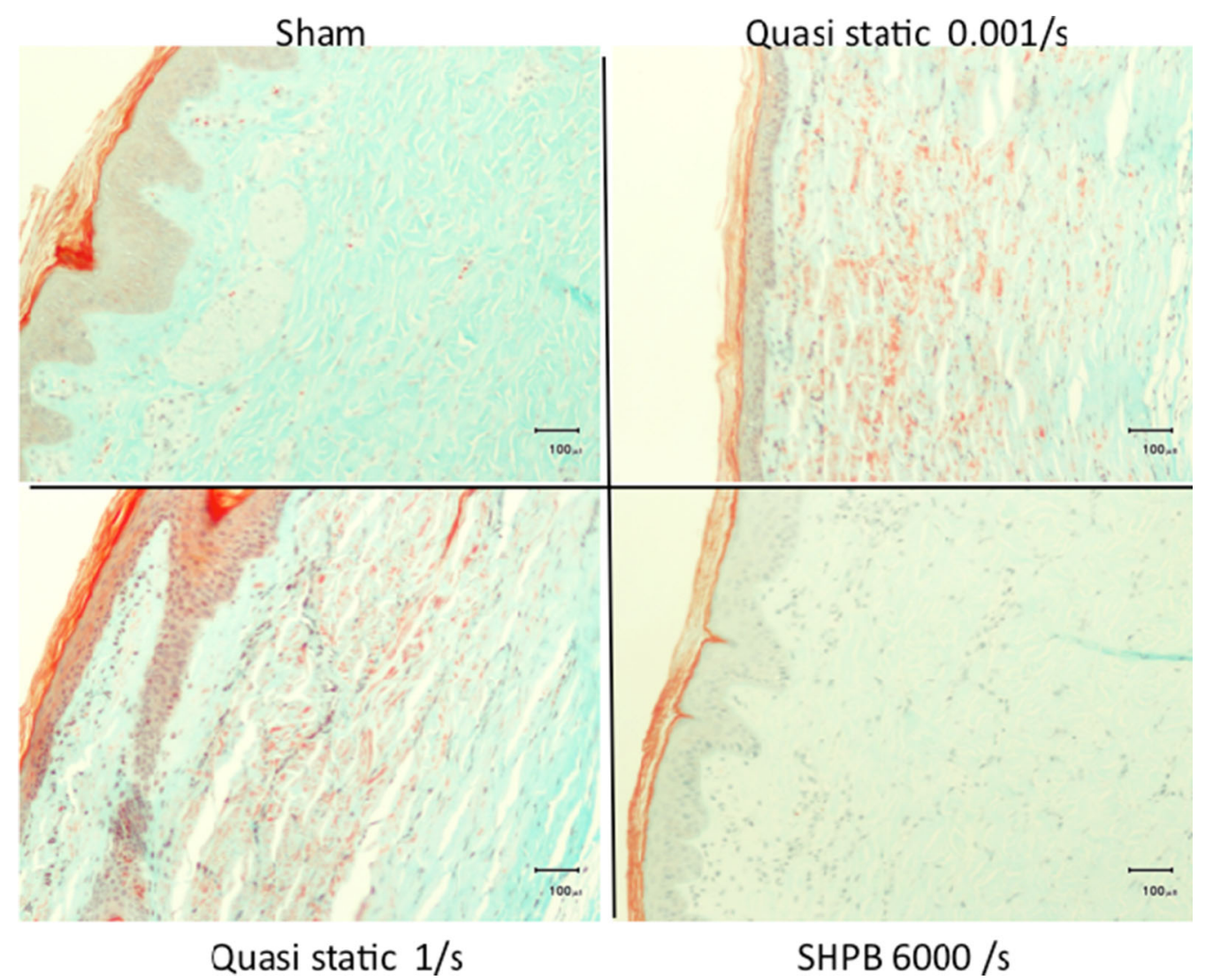

Fig. 9-Optical images of porcine rump stained with Masson's Trichrome. Instron samples are labeled "Quasi static" and a Split Hopkinson Pressure Bar sample is labeled "SHPB." The dye infiltration pattern is greater in the samples subjected to compression compared to sham. 


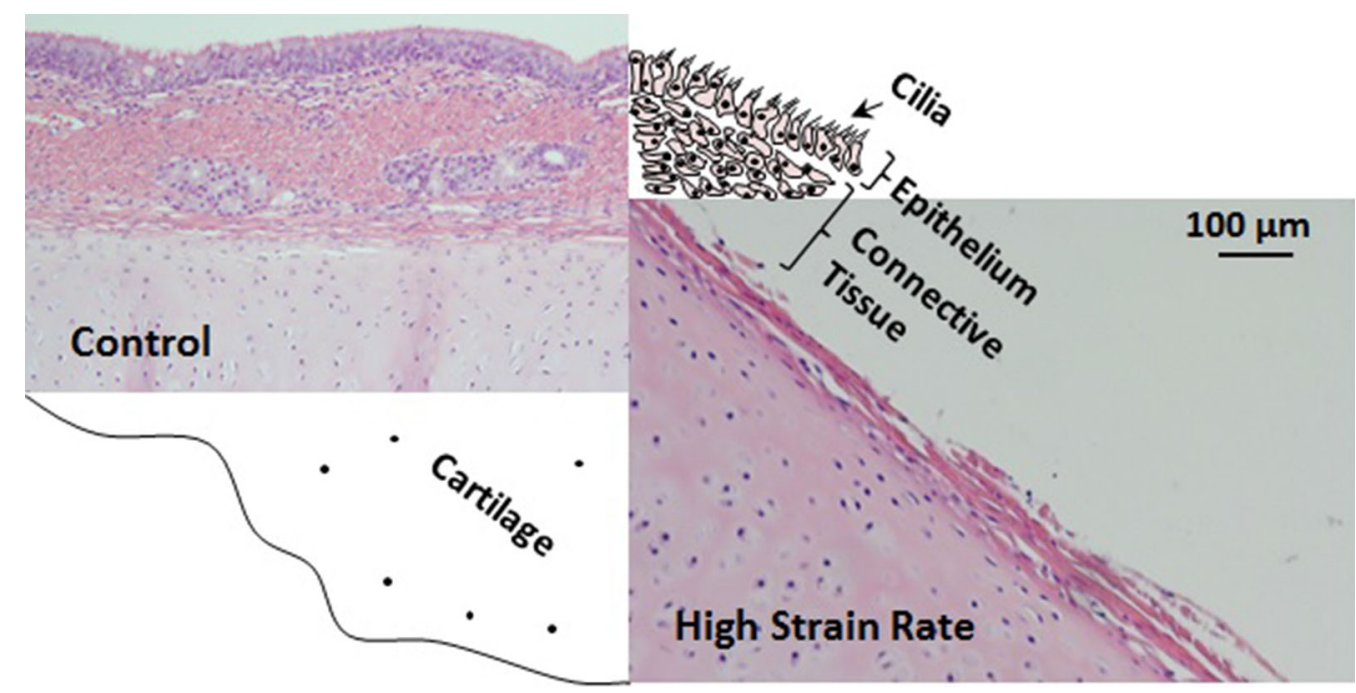

Fig. 10-Photomicrographs of a porcine trachea. A control sample is shown in the upper left quadrant and a compressed sample is shown in the lower right quadrant. Embedded schematics are included to identify the cellular structures visible in these images, from cilia located at the air-interface to cartilage. A scale bar indicating $100 \mu \mathrm{m}$ is also shown.

The increased presence of dye in slowly compressed samples indicates disruption of tissue organization, most likely due to damage of collagen structures.

As a comparison, Figure 10 shows the effects of SHPB compression of porcine trachea tissue subjected to a strain rate of $6000 \mathrm{~s}^{-1}$. These optical micrographs indicate that control samples show normal cellular architecture, identical to that observed in fresh uncompressed trachea. The outermost layer of the trachea is dominated by ciliated cells. However, the observed ablation of the cilia layer of cells and compression of the underlying connective tissue arising from the application of high strain rates indicates the relative resilience of layered structures in the trachea to blast loading. Similar to the skin studies described above, structural changes to collagen fibrils in the resilient cartilaginous layer of the trachea are most likely responsible for stiffening effects observed at high-strain rates (data not shown).

\section{Fibrous Tissue Level}

Understanding tissue behavior is vital in explaining traumatic mechanisms and injury patterns. This understanding should aid improved prevention, reconstruction, and rehabilitation of injury. In addition, in order for the behavior of a computational model to be biofidelic, accurate material models of the behavior of its components are mandatory. Whereas skeletal and soft tissue behavior is fairly well understood in slow loading-rate conditions, this is not the case in higher loading-rate conditions, such as those seen in blast. McElhaney ${ }^{[29]}$ tested bovine bone at strain rates up to $1500 \mathrm{~s}^{-1}$ and found that the modulus and strength of bone increased with strain rate. This increase is not linear; rather, it appears logarithmic suggesting that the effect diminishes at very high rates. Similarly, Mattucci et al. ${ }^{[30]}$ tested cervical spine ligaments at strain rates up to $250 \mathrm{~s}^{-1}$ and found a similar trend to that of McElhaney ${ }^{[29]}$ in bone in the variation of material

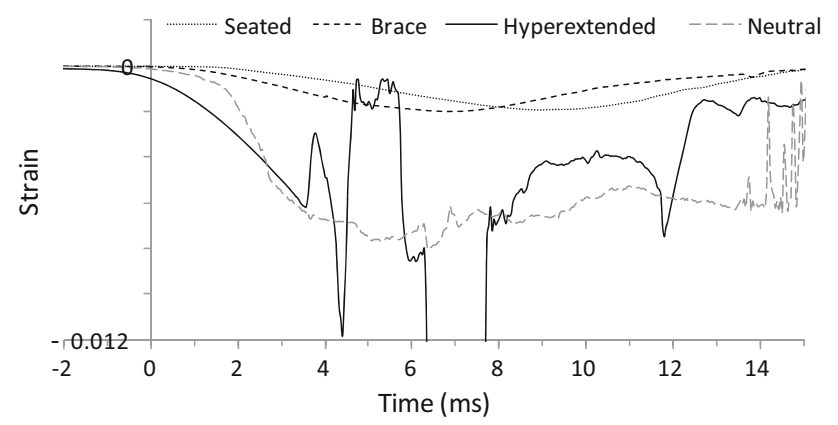

Fig. 11-Response of cadaveric legs when imparted with $500 \mathrm{~J}$ in AnUBIS.

properties with strain rate. We conducted some preliminary experiments with the lateral collateral ligament of the porcine stifle joint up to $100 \mathrm{~s}^{-1}$ and found that there appears to be a strain-sensitivity limit at approximately $1 \mathrm{~s}^{-1}$ beyond which ligament properties are virtually unaffected by strain rate. ${ }^{[31]}$

\section{Structural Level}

Traumatic injury simulators ran in the controlled environment of the laboratory offer a means of assessing injury risk and mitigation. ${ }^{[19]}$ Battlefield data on injury of the lower extremity, ${ }^{[3,18]}$ suggested that posture would affect the injury severity. AnUBIS was used to conduct experiments with cadaveric lower extremities in 4 different postures. ${ }^{[32]}$ Postures that involved flexion of the knee joint are prone to a less severe injury compared to those where the knee joint is straight.

The strain response at the heel is shown in Figure 11. In these studies, seated and braced legs do not sustain any medically significant injury, whereas standing, in either neutral or hyperextended positions sustain fractures. This is denoted by a much higher initial strain followed by a sharp reduction in the signal followed by 
increased noise. These studies were accompanied by clinical scoring of the outcome using the foot and ankle severity scale; the results show that the two standing postures sustained severely disabling injuries.

\section{CONCLUSIONS}

The study of injury to human subjects is important in a range of civilian and military scenarios. In order to study the subject a range of loading devices operating in the appropriate loading range is necessary, this requires good interaction between physical, biological, and medical sciences.

The studies outlined above feed the requirement for quantitative data required for mechanical modeling and assessing the medical outcomes of dynamic loading. A range of length and time scales is necessary, from cellular level to structural levels (e.g., legs) with loadings varying from intense micro-second duration to longer, but aggressive, second-long, pulses.

In all cases there is a need to closely analyze the results with respect to the context of biological and physical loading.

One area, not addressed here, that is of vital importance is understanding the dynamic response of lung tissues which represent a complex mix of solid, liquid, and gaseous components.

\section{ACKNOWLEDGMENTS}

This paper was presented as part of a session dedicated to Prof. Marc Meyers of the University of California, San Diego, at the Materials Society Meeting in February 2014. The Centre for Blast Injury Studies acknowledges the support of the Royal British Legion and Imperial College London. The Institute of Shock Physics acknowledges the support of the Atomic Weapons Establishment, Aldermaston, UK and Imperial College London.

\section{REFERENCES}

1. B.D. Owens, J.F. Kragh, Jr., J. Macaitis, S.J. Svoboda, and J.C. Wenke: J. Orthop. Trauma, 2007, vol. 21, pp. 254-57.

2. A. Ramasamy, S. Harrisson, I. Lasrado, and M.P. Stewart: Injury, 2009, vol. 40, pp. 493-97.

3. A. Ramasamy, A.M. Hill, S.D. Masouros, I. Gibb, A.M.J. Bull, and J.C. Clasper: J. R. Soc. Interface, 2011, vol. 8, pp. 689-98.

4. W.G. Proud: J. R. Army Med. Corps, 2013, vol. 159, pp. i4-9.

5. M.A. Meyers: Dynamic Behavior of Materials, Wiley, New York, 1994.
6. J.A. Singleton, I.E. Gibb, N.C. Hunt, A.M.J. Bull, and J.C. Clasper: BMJ Open, 2013, vol. 3, p. e003130.

7. J.E. Risdall and D.K. Menon: Philos. Trans. R. Soc. Lond. B Biol. Sci., 2011, vol. 366, pp. 241-50.

8. K.A. Alfieri, J.A. Forsberg, and B.K. Potter: Bone Joint Res., 2012, vol. 1, pp. 192-97.

9. S.D. Masouros, K.A. Brown, J. Clasper, and W.G. Proud: Proc. ICE Eng. Comput. Mech., 2013, vol. 166, pp. 113-18.

10. T.-T.N. Nguyen, T. Davey, and W.G. Proud: Proceedings of New Trends in Research of Energetic Materials, Pardubice, Czech Republic, 2014, pp. 351-58.

11. M. Rogers: Proceedings of the 11th International Symposium in Shock Tubes and Shock Waves, University of Washington Press, Seattle, 1977.

12. G. Kinney and K. Graham: Explosive Shock in Air, Springer, Berlin, 1985.

13. C. Bo, N. Newell, T.-T. Nguyen, B. Butler, J. Wilgeroth, J. Balzer, S. Masouros, A. Bull, A. Phillips, A. Jardine, A. Williams, S. Rankins, K. Brown, and W. Proud: Proceedings of New Trends in Research of Energetic Materials, Czech Republic, 2013.

14. L. Sadwin and M. Swisdak, Jr.: Proceedings of New Trends in Research of Energetic Materials, Czech Republic, 2014.

15. T.-T. Nguyen, J. Wilgeroth, and W. Proud: Proceedings of 18th Biennial International Conference of the APS Topical Group on Shock Compression of Condensed Matter held in conjunction with the 24th Biennial International Conference of the International Association for the Advancement of High Pressure Science and Technology (AIRAPT), Seattle, 2014.

16. P. Cooper: Explosives Engineering, Wiley-VCH, New York, 1997.

17. T.-T. Nguyen, T. Davey, and W. Proud: Proceedings of New Trends in Research of Energetic Materials, Pardubice, Czech Republic, 2014.

18. A. Ramasamy, A.M. Hill, and S.D. Masouros: J. Bone Joint Surg., 2013, vol. 95, pp. e25-27.

19. S.D. Masouros, N. Newell, and A. Ramasamy: Ann. Biomed. Eng., 2013, vol. 41, pp. 1957-67.

20. D.R. Richmond and C.S. White: The Symposium on Effectiveness Analysis Techniques for Non-nuclear Warheads Against Surface Targets, Dahlgren, USA, 1962.

21. C.R. Bass, K.A. Rafaels, and R.S. Salzar: J. Trauma, 2008, vol. 65, pp. $604-15$.

22. C. Bo, A. Williams, S. Rankin, W.G. Proud, and K.A. Brown: J. Phys., 2014. DOI:10.1088/1742-6596/500/10/102001.

23. C. Bo, J. Balzer, K.A. Brown, S.M. Walley, and W.G. Proud: Eur. Phys. J. Appl. Phys., 2011, vol. 55, pp. 1-5.

24. C.A. Cipriano, S.G. Pill, and M.A. Keenan: J. Am. Acad. Orthop. Surg., 2009, vol. 17, pp. 689-97.

25. W.M. Jackson, A.B. Aragon, J.D. Bulken-Hoover, L.J. Nesti, and R.S. Tuan: J. Orthop. Res., 2009, vol. 27, pp. 1645-51.

26. M. Delius: Shock Waves, 1994, vol. 4, pp. 55-72.

27. T. Kodama, M.R. Hamblin, and A.G. Doukas: Biophys. $J$., 2000, vol. 79 , pp. $1107-22$.

28. G.T. Christopherson and L.J. Nesti: Stem Cell Res. Ther., 2011, vol. 2 , p. 40.

29. J.H. McElhaney: J. Appl. Physiol., 1966, vol. 21, pp. 1231-36.

30. S.F. Mattucci, J.A. Moulton, N. Chandrashekar, and D.S. Cronin: J. Mech. Behav. Biomed. Mater., 2012, vol. 10, pp. 216-26.

31. T.J. Bonner, N. Newell, A.D. Pullen, A.M.J. Bull, and S.D. Masouros: Proceedings of the International Research Council on the Biomechanics of Injury Conference, 2013, vol. 134.

32. S.D. Masouros, N. Newell, T.J. Bonner, A. Ramasamy, A.M. Hill, A.T.H. West, J.C. Clasper, and A.M.J. Bull: Proceedings of the IRCOBI Conference in Dublin, Ireland, 2012. 